M.Sc. Mauricio Monge A. mmonge@itcr.ac.cr Calle 6 Ave 11 Instituto Tecnológico de Costa Rica Cartago - Costa Rica.

Estudiante Programa de Doctorado "Economía y Gestión de la Empresa" Departamento de Economía de la Empresa, Universidad Politécnica de Cartagena (UPCT). Magister Scientae en computación, Mención Honorífica: Magna

Cum Laude y Licenciado en Administración de Empresas del Instituto Tecnológico de Costa Rica.

Antonio J. Briones Peñalver Ph.D. aj.briones@upct.es Calle Real, 3-30201 Universidad de Cartagena Cartagena - España.

Doctorado con Acreditación Europea. Doctorado Europeo en Economía y Gestión de Empresas, por la Universidad Politécnica de Cartagena, España. Premio Extraordinario de Doctorado en la Universidad

Politécnica de Cartagena, por

la Facultad de Ciencias de la Empresa en el curso 2007/2008.

Domingo García Pérez de Lema Ph.D. domingo.garcia@upct.es Calle Real, 3-30201 Universidad de Cartagena Cartagena - España.

Doctor en Ciencias Económicas y Empresariales, Universidad de Murcia - España. Premio Extraordinario de Doctorado en la Universidad de Murcia, Facultad en Ciencias Económicas y Empresariales curso $1988 / 1989$.

Artículo de investigación científica o tecnológica Según Clasificación Colciencias

Fecha de recepción: julio 18 de 2011 Fecha de corrección: octubre 28 de 2011 Fecha de aprobación: diciembre 20 de 2011

\section{Factores determinantes de la creación de las Spin Off académicas: caso del Instituto Tecnológico de Costa Rica}

Determining factors of the creation of academic Spin Offs: case of the Instituto Tecnológico of Costa Rica

Facteurs déterminants de la création des Spin Off académiques: cas de l’institut technologique de Costa Rica

\section{Resumen}

Este trabajo tiene como objetivo analizar los factores determinantes en la creación de Spin off académicas en el Instituto Tecnológico de Costa Rica (ITCR), universidad pública de Costa Rica, utilizando la metodología propuesta por 0'Shea et al., (2008): atributos del emprendedor, recursos organizativos, determinantes institucionales y ambientales. Los resultados relevan que en el ITCR la principal fortaleza es su misión, cultura e historia, caracterizada por el servicio brindado al sector productivo; no obstante, requiere fortalecer aspectos como la calidad de la investigación y consecución de fondos externos para esta, así como aspectos externos de índole legal que brinden las condiciones apropiadas para la creación de spin off académicas, y la generación de capital de riesgo. Todo ello, a tono con el paradigma de la universidad emprendedora. Este artículo contribuye a explicar el proceso de creación de Spin off en una universidad pública latinoamericana y es de utilidad tanto para el ámbito académico, como público y privado encargado de impulsar un mayor acercamiento entre la Universidad y la Sociedad.

Palabras clave: emprendedurismo académico, universidad emprendedora, spin off académico. 


\section{Determining factors of the creation of academic Spin Offs: case of the Instituto Tecnológico of Costa Rica}

\section{Factores determinantes de la creación de las Spin Off académicas: caso del Instituto Tecnológico de Costa Rica}

\section{Facteurs déterminants de la création des Spin Off académiques : cas de l'institut technologique de Costa Rica}

\section{Abstract}

This work seeks to analyze the determining factors in the creation of academic spin offs in the Instituto Tecnológico of Costa Rica (ITCR), a public university of Costa Rica, by using the methodology proposed by O'Shea et al., (2008): attributes of the entrepreneur, organizational resources, institutional and environmental determinants. The results reveal that in the ITCR the main strengths are its mission, culture, and history characterized by the service offered to the production sector; nevertheless, it needs to enhance aspects like the quality of research and secure external funds for said research, as well as external legal aspects that offer the appropriate conditions for the creation of academic spin offs and the generation of risk capital; all this, in keeping with the paradigm of the entrepreneurial university. This article contributes to explain the process of creating spin offs in a Latin American public university and it is useful for the public and private academic sector in charge of promoting greater closeness between the University and Society.

Keywords: academic entrepreneurship, entrepreneurial university, academic spin off.
Facteurs déterminants de la création des Spin Off académiques : cas de l'institut technologique de Costa Rica

\author{
Factores determinantes de la creación de \\ las Spin Off académicas: caso del Instituto \\ Tecnológico de Costa Rica
}

\author{
Determining factors of the creation of \\ academic Spin Offs: case of the Instituto \\ Tecnológico of Costa Rica
}

\section{Résumée}

Ce travail a pour objectif l'analyse des facteurs déterminants de la création des Spin off académiques dans l'Institut Technologique de Costa Rica (ITCR), connu aussi comme l'université publique de Costa Rica. Cette analyse a été faite à partir de la méthodologie proposée par O־Shea et al. (2008) : attributs de l'entrepreneur ; ressources organisationnelles; déterminants institutionnels et de l'environnement.

Les résultats démontrent qưà l'intérieur de l'ITCR le principal point fort repose sur sa mission, sa culture et son histoire, et se caractérisé par le service que l'Institut fournit au secteur productif. Cependant il faut travailler sur le renforcement d'aspects tels que la qualité de la recherche et la consécution de ressources externes pour celle-ci ; de même, sur des aspects externes de type légal pour garantir les conditions convenables pour la création de Spin off académiques et la génération de capital de risque. Tout cela en correspondance avec le paradigme de l'université entrepreneuriale. Cet article contribue avec l'explication du processus de création de Spin off dans une université publique en Amérique Latine, et il résulte utile pour le domaine académique tant au niveau publique qúau niveau privé. Ce dernier étant chargé de resserrer davantage les liens entre l'Université et la société.

Mots clef: entrepreneuriat académique, université entrepreneuriale, spin off académique. 


\section{Factores determinantes de la creación de las Spin Off académicas: caso del Instituto Tecnológico de Costa Rica}

\section{Introducción}

Los gobiernos y sus universidades dan importancia a la creación de empresas universitarias como instrumento para la comercialización de la investigación (Shane, 2004; Wright, Hmieleski, Siegel, \& Ensley, 2007), preparando oficinas de transferencia de tecnología, incubadoras de empresas y fondos internos de capital semilla para la comercialización de la investigación (Rasmussen, Moen, \& Gulbrandsen, 2006).

El creciente interés en las spin off universitarias entre los tomadores de decisiones y la gran cantidad de recursos destinados para apoyarlas (Lockett, Siegel, Wright, \& Ensley, 2005) justifica investigar cómo las universidades pueden facilitar la creación de nuevas empresas basadas en la investigación (Markman, Gianiodis, \& Phan, 2008)

Las spin-off son empresas basadas en el conocimiento científico y tecnológico desarrollado por docentes e investigadores o estudiantes de doctorado, de las universidades. Se consideran como el mejor instrumento para la transferencia de la investigación a la sociedad, por ser innovadoras, altamente competitivas y de gran aceptación en el mercado (ANCES, 2003).

La importancia de estudiar la creación del spin-off académica, radica en su capacidad de impactar positivamente en el mercado, con un nuevo producto o servicio innovador, de valor agregado resultado del conocimiento científico y tecnológico de la universidad, transferido al mercado para su comercialización (Casanova, 2004; 0'Shea, et al., 2007; Laborda \& Briones, 2010).

En una economía globalizada y altamente competitiva, la salud económica y social de cualquier sociedad depende de su capacidad de incorporar conocimiento científico y tecnológico (Beraza \& Rodríguez, 2010). Así, surge un sistema de innovación que vincula la ciencia y la tecnología con el desarrollo socioeconómico, apoyado en la interacción de universidades e instituciones de investigación, las empresas y el gobierno (Etzkowitz \& Leydesdorff, 2000).
Para Costa Rica, esto constituye una oportunidad hacia su desarrollo económico, científico y social, razón que justifica investigar el comportamiento de los factores determinantes del proceso de creación de spin off académicas.

El objetivo principal es exponer el caso del Instituto Tecnológico de Costa Rica, la identificación y caracterización del proceso de creación de spin-off en esta universidad, los factores determinantes -características institucionales, recursos organizacionales, factores ambientales y las características del emprendedor académico- que inciden en la creación y evolución de las empresas. Se utilizó la metodología cualitativa análisis de caso y se realizaron entrevistas personales, a los emprendedores académicos identificados, autoridades universitarias y funcionarios de gobierno, a través de un cuestionario, de acuerdo a los trabajos realizados por 0' Shea et al., (2007) y Aceytuno \& Paz (2008).

\section{Proceso de creación de spin-off académicas}

$0^{-}$Shea et al., (2008), estructuran los cuatro factores tomados en cuenta en esta investigación, como "determinantes de la creación de spin-off en el contexto universitario", a saber: (1) los atributos y las características de personalidad de los emprendedores académicos; 2) los recursos propios y las capacidades de la universidad; 3) la estructura universitaria y las políticas de comercialización; y 4) los factores ambientales que influyen en los emprendimientos académicos. Nlemvo et al., (2002); Vohora et al., (2004); Lockett \& Wright (2005) y 0'shea et al., (2007) avalan la importancia de estos cuatro factores como los principales del proceso de creación de spinoff acádemicos.

Se observa en la Figura 1 que a partir de la identificación de la oportunidad tecnológica (Roberts, 1991), se identifican varios grupos de factores determinantes que influyen en la decisión de crear la spin-off y su proceso de formación. 
La primera categoría de factores determinantes incluye los atributos y la personalidad del empresario (Roberts, 1991); la segunda los recursos organizativos de la universidad (Blumenthal, Campbell, Causiono, \& Louis, 1996; Zucker, Darby, \& Brewer, 1998; Di-Gregorio \& Shane, 2003; Lockett \& Wright, 2005; O'Shea, Allen, \& Morse, 2005; Powers \& McDougall, 2005 ; Stuart \& Ding, 2006); la tercera los determinantes institucionales (estructuras y políticas que facilitan la comercialización) (Louis, Blumenthal,
M.Gluck, \& Stoto, 1989; Henrekson \& Rosenberg, 2001; Feldman, Feller, Bercowitz, \& Burton, 2002; Nlemvo, Pirnay, \& Surlemont, 2002; Di-Gregorio \& Shane, 2003; O'Shea et al., 2004; Link \& Scott, 2005; Lockett, et al., 2005; Djokovic \& Souitaris, 2008; Markman, et al., 2008; Martinelli, Meyer, \& von Tunzelmann, 2008); y la cuarta los factores externos o ambientales (Florida \& Kenney, 1988; Sorenson \& Stuart, 2001 ; Wright, Birley, \& Mosey, 2004; Powers \& McDougall, 2005 ; O’Shea, et al., 2007).

\section{Figura 1. Factores determinantes de la generación de Spin-offs académicas}

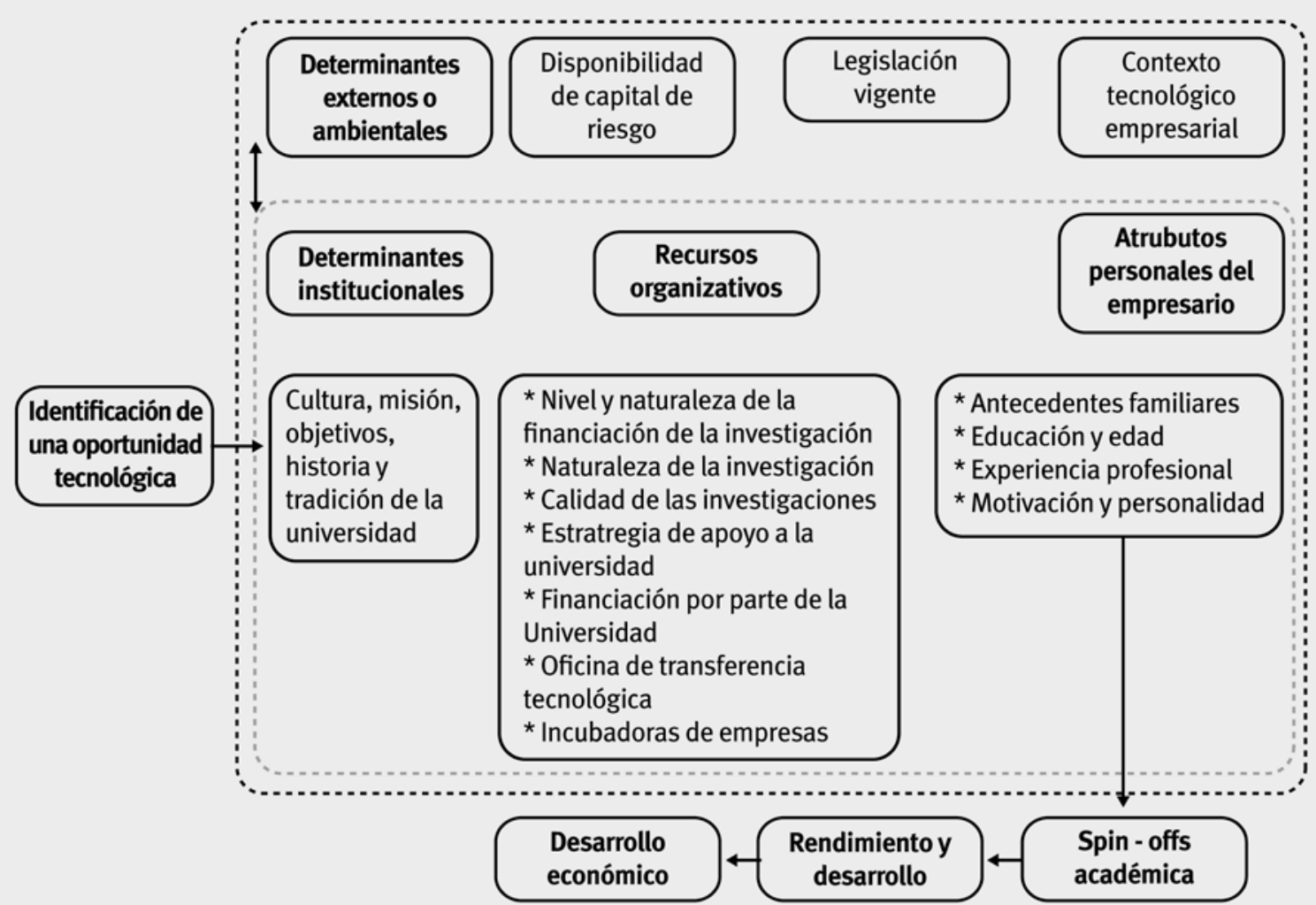

Fuente: Aceytuno y Paz (2008).

\subsection{El emprendedor académico como determinante}

Este determinante es clave en el proceso de creación de las spin-off académicas. Los estudios en este campo coinciden en que la base de la creación de spin-off, se deben a los factores personales del investigador (o investigadores), tales como la personalidad, habilidades, trayectoria profesional y disposición a implicarse en actividades empresariales (Aceytuno \& Paz, 2008). Este factor determinante, dada su magnitud e importancia, se analizará en un estudio posterior.

\subsection{Recursos organizativos de la universidad}

Los recursos disponibles en cada universidad, fruto de su historia y éxitos pasados, es un determinante en el número de spin-off generadas y permite explicar las diferencias, en este aspecto, entre universidades (Aceytuno \& Paz, 2008). Entre estos tenemos:

a) El nivel y naturaleza de los fondos que se utilizan para financiar la investigación. Blumenthal et al.,(1996); Powers \& McDougall, 
(2005) y 0`Shea et al., (2004) plantean que la procedencia y cuantía de los fondos utilizados para financiar la investigación son un factor determinante en la generación de spin-off, ya que la investigación financiada con recursos privados es más susceptible de ser comercializada, así los investigadores cuya actividad es financiada mediante recursos procedentes de la industria, son más activos en la transferencia comercial de los resultados, tanto mediante el uso de licencias de patentes, como a través de la generación de spin-off (Aceytuno \& Paz, 2008);

b) La naturaleza de la investigación, que incluye tanto el nivel de oportunidades, como la propensión a comercializar los resultados de la investigación. Varían considerablemente entre los distintos campos científicos (Shane, 2004; Siegel \& Phan, 2006) y son más proclives en aquellos sectores de actividad científica y tecnológica (Fontes, 2005). 0’Shea et al., (2004) señalan que la financiación de investigaciones en ciencias e ingeniería es más fructífera en generación de spin-off, destacando especialmente las investigaciones que se llevan a cabo en las ramas de ciencias de la salud, la informática y la química;

c) La calidad de los investigadores. Influye positivamente en la obtención de resultados importantes, que pueden ser comercializados mediante la creación de una spin-off (0'Shea, et al., 2004; Powers \& McDougall, 2005 ). Los trabajos de Powers \& McDougall (2005) y O’Shea et al.,(2004) mencionan que la calidad de la investigación en la universidad es un factor determinante en la generación de spin-off. Al obtener resultados importantes y la participación de los científicos y universidades de prestigio, favorece la comercialización, a través de la creación de empresas. La calidad de los investigadores es medida a través de varios índices COTEC (2006): competitividad investigadora medida como porcentaje de proyectos de $I+D$ aprobados sobre los presentados y esfuerzo investigador medido como el porcentaje de proyectos de I+D aprobados por profesor en nómina. Zucker et al., (1998) destacan tanto la importancia de la presencia de "científicos estrella" y sus colaboradores para la generación de spin-off, como su relación con universidades de prestigio, considerando que éstos crean las spin-off con el objetivo de obtener rentas de su capital intelectual. Di Gregorio y Shane señalan que existe una relación positiva entre la generación de spin-off y el prestigio de las universidades, basándose en que explotar tecnologías cuyo resultado comercial es incierto, es más fácil cuando el prestigio de la universidad respalda la credibilidad del emprendedor académico:

d) La estrategia de apoyo a la generación de spin-off académica, relacionada con la cultura y los objetivos de la universidad. Clarysse et al., (2005) diferencian tres modelos: i) modelo de selección baja, cuyo objetivo es generar un mayor número de spin-off, tanto de alumnos como de investigadores, sin darle importancia al aspecto económico y financiero. ii) modelo de apoyo, que considera la generación de spin-off como una forma de comercializar los resultados de la investigación, de manera alternativa a la licencia de patentes. iii) modelo de la incubadora, cuyo objetivo es buscar oportunidades derivadas de la investigación científica, donde la spinoff es la forma más beneficiosa de explotación comercial, frente al licenciamiento de patentes;

e) La disponibilidad de oficinas de transferencia tecnológica en la universidad. Se puede considerar un factor determinante del surgimiento y desarrollo de spin-off universitarias (Lockett, et al., 2005; 0'Shea, et al., 2008; O'Gorman, Byrne, \& Pandya, 2008 ) que son creadas para mediar entre los investigadores de la universidad y la industria. Sus funciones incluyen la promoción de las spin-off y la difusión de los resultados de la investigación que se lleva a cabo (Siegel, Waldman, \& Link, 2003), así como la gestión del valor de la propiedad intelectual (Meseri \& Maital, 2001) o colaborar con los investigadores en la difusión de los resultados de su investigación (Carlsson \& Fridh, 2002 ; Jain \& George, 2007). Destacan las siguientes funciones (Roberts \& Malone, 1995): i) Toma de decisiones durante el proceso de evaluación de las posibilidades comerciales del invento; ii) Planificar la protección intelectual del invento; iii) Relacionar a los empresarios con empresas de capital riesgo; iv) Participar en los organismos gestores de la empresa. Estudios han analizado la influencia de las oficinas de transferencia sobre la generación de spin-off y su efectividad como mecanismo de transferencia (Parker \& Zilberman, 1993; Jain \& George, 2007). Sin embargo, no existe consenso sobre su efectividad en la genera- 
ción del spin-off, aunque su influencia se considera generalmente positiva (0'Shea et al., 2007). Powers y McDougall (2005) indican que la efectividad de las mismas está relacionada con su antigüedad, mientras que otros autores destacan que, en ocasiones, los empresarios no están satisfechos con el asesoramiento recibido (Siegel et al., 2003). No obstante constituye un elemento más de apoyo en una actividad compleja e influenciada por varios factores, como por ejemplo, la influencia del entorno empresarial (Debackere, 2000); siendo importante también considerar el contexto social e institucional dentro del cual se desenvuelven estas oficinas (Jain \& George, 2007); g) Por último, la presencia de incubadoras de empresas. Son centros en los que se sitúan las spin-off durante sus primeros años de actividad (Rogers et al., 2001; Clarysse et al., 2004; Rothaermel y Thursby, 2005). Steffensen et al., (1999) consideran que son útiles en las primeras etapas de funcionamiento de la spin-off y cuando existen fuertes relaciones entre los investigadores y la universidad (Rothaermel y Thursby, 2005). Otras investigaciones muestran que, la influencia de las incubadoras universitarias sobre la generación de spin-off, es insignificante, aunque no implica que no puedan favorecer su éxito (Di Gregorio y Shane, 2003).

\subsection{Determinantes institucionales}

Louis et al., (1989), Henrekson y Rosenberg, (2001); Feldman et al., (2002); Ndonzuau, (2002); Di Gregorio y Shane, (2003); Link y Scott, (2005); Lockett y Wright, (2005 a); O'Shea et al., (2004); Djokovic y Souitaris, (2008); Martinelli et al., (2007); Markman et al., (2008) han tratado estos determinantes y su importancia, identificando a tres como los principales: la misión de la universidad, la cultura y su historia y la tradición (Kenney \& Goe, 2004; Kirby, 2006; 0'Shea, et al., 2007), permitiendo identificar a las universidades que se encuentran dentro del paradigma de la "universidad emprendedora", en las que estos factores se encuentran orientados hacia la comercialización de los resultados de la investigación y la creación de empresas, como son el MIT o la Universidad de Stanford en EE.UU (Kenney \& Goe, 2004; O'Shea, et al., 2007).

Existen otras universidades en cuya cultura continua predominando el "paradigma científico" (Feldman y Desrochers, 2004), donde es común que la universidad evite la implicación de los científicos en los usos últimos de la investigación, y no se favorece ningún tipo de transferencia tecnológica, y menos aun, la creación de empresas por parte de los investigadores. Ndonzuau et al.,(2002) señalan que el paradigma científico ha contribuido al establecimiento de un sistema de recompensas en la universidad, en el que se identifican tres características que no favorecen la transferencia tecnológica: la estrategia de "publicar o morir", la ambigüedad de la relación de los investigadores con el dinero y la naturaleza desinteresada de la investigación académica. Por otro lado, Kirby (2006) señala que la naturaleza impersonal de las relaciones en la universidad, su estructura jerárquica, el conservadurismo o la inexistencia de métodos de compensación apropiados, pueden ejercer un efecto desincentivador de la actividad empresarial.

\subsection{Determinantes externos 0 ambientales}

Impulsan o no, la creación de spin-off académicas. Su importancia radica en que son condicionantes del contexto; un contexto favorable, que incluya i) La existencia de empresas de capital riesgo; ii) La entrada en vigor de leyes que favorezcan la creación de spin-off; y iii) La situación de la universidad en un contexto tecnológico y empresarial, promoverá la aparición de spin-off académicas (Aceytuno y Paz, 2008).

i) Las empresas de capital riesgo financian las nuevas empresas a cambio de participaciones en el capital social de las mismas (Florida y Kenney, 1988), financiando proyectos que por diversas causas, como son el ser un productor innovador, o tener un alto índice de riesgo, o ser de pequeño tamaño, entre otros, no pueden acudir a las fuentes de financiación tradicionales, a un coste aceptable (Sarasa, 1986). Martín (1988) define al capital de riesgo como "un sistema de financiación dirigido esencialmente a pequeñas y medianas empresas, mediante el cual una sociedad especializada en inversiones inyecta capital en una pequeña o mediana empresa en una proporción minoritaria y por un espacio de tiempo relativamente corto". El capital de riesgo aporta, en la mayoría de casos, fondos propios en lugar de deuda, lo 
que no sólo mejora la imagen de la empresa, sino que posibilita la posterior obtención de nueva financiación mediante endeudamiento. Su presencia en la empresa no es permanente ya que abarca un periodo que oscila entre cuatro y siete años, obteniendo los beneficios en forma de plusvalía una vez efectuada la desinversión; donde además de los fondos, las entidades de capital riesgo aportan también un valor intangible materializado en su experiencia, contactos, ayuda y otros (Ramón \& García, 2004, 2006). Se procura una reducción en el riesgo en la inversión, mediante una selección cuidadosa de los proyectos a financiar y una participación activa en la gestión de la nueva empresa (Florida y Kenney, 1988); considerando, además, que la disponibilidad de fondos de capital de riesgo, influye positivamente en la generación de spin-off (Powers \& McDougall, 2005 ). Wright et al., (2004) señalan la importancia del acceso al capital de riesgo, destacando que la obtención de este tipo de financiación es más eficaz para las spin-off universitarias, que toman la forma de "joint venture". Sorenson y Stuart (2001) introducen la variable geográfica y señalan que la probabilidad de que una empresa spin-off reciba financiación de capital riesgo, se reduce a medida que la distancia entre ambas es mayor. Di Gregorio y Shane (2003) consideran al capital de riesgo externo como un factor determinante en la generación de spin-off universitaria y apuntan que el capital de riesgo que proporciona la universidad no genera efecto alguno, fundamentándolo en que el capital de riesgo universitario únicamente supone un sustituto del capital externo en la generación del spin-off.

ii) La legislación vigente en materia de universidades y propiedad intelectual se considera una variable importante para la generación de spin-off. Shane, (2004) señala que la entrada en vigor de la Bayh-Dole Act en EEUU -ley de 1980 que concede a los centros de investigación los derechos de propiedad intelectual sobre los resultados de la investigación financiada con fondos públicos- tuvo efectos positivos sobre la generación de spinoff universitarias. La legislación española, a través de la Ley Orgánica Universitaria (LOU) y sus reformas en el año 2007, fomenta la interrelación entre la universidad y la industria (“Ley Orgánica 4/2007," 2001). En el anterior marco normativo español, la creación de empresas por parte del personal de una universidad se encontraba muy limitado, dada la incompatibilidad existente entre ser partícipe en una empresa privada y el trabajo en la universidad. La Reforma a la LOU elimina esta limitación y establece, entre otras, la posibilidad de que los profesores funcionarios de las universidades públicas, disfruten de un quinquenio sabático para crear una spin-off académica, conservando su plaza en la universidad. Golfard y Henrekson, (2003) señalan el posible efecto desincentivador de la creación de spin-off académicas que puede tener la legislación sueca, pues no se ha logrado crear un contexto que incentive la comercialización de los resultados por parte de los investigadores. Bacchiocchi y Montobbio (2007) coinciden en señalar la cautela que deben tener los políticos, al diseñar las políticas de innovación, especialmente al intentar imitar las legislaciones de patentes vigentes en EEUU, como la Bayh-Dole Act, sin tomar en cuenta el contexto local.

iii) Finalmente, otro de los factores externos que puede influir sobre la generación de spin-off académica es el contexto tecnológico y empresarial. Algunos autores destacan que el surgimiento de spin-off en una universidad que se encuentre localizada en un entorno altamente tecnológico y emprendedor, será mayor que en otras universidades sin este entorno (Roberts, 1991; Saxenian, 1994; Jong, 2006; 0`Shea et al., 2007). El diseño de una política de fomento de las spin-off académicas, se debe basar en un análisis cuidadoso del contexto y de las instituciones que trabajan en el mismo (Aceytuno y Paz, 2008).

\section{Metodología de la investigación}

La investigación se circunscribe al Instituto Tecnológico de Costa Rica, en su sede central del campus Cartago, Costa Rica y a los aspectos identificados como generadores de spin off académicas, por cuanto se contaba con mayor información para realizarla.

Se utilizó el método del caso en esta investigación, apoyándose en el marco teórico desarrollado por 0`Shea (2005), con el análisis de las variables involucradas, como un primer acercamiento a la realidad objeto de estudio (Maxwell, 1998), buscando respuesta a la interrogante ¿Cuál es el comportamiento 
de los factores determinantes que incidieron en la creación y evolución de las spin off académicas identificadas en el ITCR?

Como medio de comparación se utilizó el estudio (Yin, 2009) "La creación de spin-off universitarias: el caso de la Universidad de Huelva planteado por Aceytuno y Paz (2008)", hasta donde fue posible la equivalencia de los conceptos.

Se realizó una revisión bibliográfica sobre el estado del arte en emprendedurismo académico, así como un análisis documental de planes, programas y políticas de investigación y extensión en el ITCR.

Se realizaron entrevistas a funcionarios del sector gobierno y universitario de ciencia y tecnología de Costa Rica; quienes reconocieron la importancia del tema. No obstante, en ninguna de sus dependencias se han realizado estudios referentes al tema de esta investigación.

Por último, se llevó a cabo un análisis de la información recogida a través de entrevistas y fuentes secundarias y con base en esta información se conformó el caso empírico acerca de los factores determinantes de la creación de spin-off académicas en el Instituto Tecnológico de Costa Rica.

\section{Resultados: descripción del caso}

El Instituto Tecnológico de Costa Rica fue fundado en 1971, mediante ley No. 4777 de la Asamblea Legislativa de Costa Rica, siendo la primera y única universidad de corte tecnológico creada hasta ese momento, con la misión de "Contribuir al desarrollo integral del país, mediante la formación de recursos humanos, la investigación y la extensión; manteniendo el liderazgo científico, tecnológico y técnico, la excelencia académica y el estricto apego a las normas éticas, humanísticas y ambientales desde una perspectiva universitaria estatal de calidad y competitividad a nivel nacional e internacional" (Gaceta, 1999)

Queralt (2008) reconoce la importancia que da el ITCR, a la vinculación universidadempresa, impulsada por sus fines y principios, lo que se ha plasmado a través de cursos de capacitación, prácticas de especialidad, investigación contratada y servicios repetitivos, entre otros, realizados a través de instancias que tienen a cargo el promover la vinculación universidad-empresa como lo es el Centro de Vinculación Universidad-Empresa; el Centro de Incubación de Empresas (CIE-TEC); el Programa de Emprendedores -orientado a apoyar la formación de profesionales líderes, capaces de crear y desarrollar proyectos exitosos, así como de descubrir y apoyar a aquellos estudiantes interesados en la creación de su propias fuentes de ingreso, mediante la formación de nuevas empresas que tengan como base de su competencia los factores tecnológicos; la Fundación Tecnológica de Costa Rica (FUNDATEC): canal que facilita la prestación de servicios remunerados al sector público y privado; multiplicando el impacto en los sectores científico, tecnológico y educativo y permitiendo al ITCR generar ingresos adicionales que son utilizados en el financiamiento de nuevos proyectos de investigación y extensión.

Como resultado de la investigación bibliográfica y de campo realizada, se tiene que este es el primer estudio sobre spin-off universitarias que se realiza en Costa Rica, lo que lo convierte en un aporte significativo que permite establecer puntos de comparación, análisis estadísticos, proyecciones y modelos para la creación de spin-off académicas, entre otros.

A continuación se presentan los resultados obtenidos con respecto a los factores determinantes "recursos organizativos de la universidad", "recursos institucionales" y por último, "factores externos o ambientales". Como se mencionó anteriormente, el factor determinante "emprendedor académico", no se incluye en este trabajo y se deja para un análisis posterior, dada su magnitud e importancia.

\subsection{Recursos organizativos de la universidad}

En el caso del Instituto Tecnológico de Costa Rica, los recursos para la investigación están compuestos por el "Fondo de la Vicerrectoría de Investigación y Extensión (VIE)", integrado por recursos públicos provenientes del Estado y fondos externos que incluyen aportes de empresas privadas, organismos 
internacionales y organizaciones no gubernamentales. Los datos de años 2006 al 2008, de fondos públicos y externos, indican que en promedio, los fondos públicos para la investigación son prácticamente un $80 \%$ versus un $20 \%$ provenientes de fuentes externas. Este $20 \%$ de fondos externos para investigación en el ITCR, es muy inferior, en comparación con el $\%$ de fondos externos recibidos por la Universidad de Huelva, ya que para el año 2006, dicha universidad tuvo un aporte privado de un $50 \%$. Aceytuno y Paz (2008), refiriéndose a la Universidad de Huelva, señalan que "el nivel de los fondos que se utilizan para financiar la investigación es reducido, perjudicando tanto a la consecución de resultados como a la comercialización de los mismos, en este caso a través de la creación de spin-off universitarias". Por lo tanto, se puede decir que las condiciones del ITCR, en comparación con la Universidad de Huelva, son aún más difíciles para el surgimiento de spin-off académicas.

\subsubsection{Calidad de la investigación en el ITCR}

El ITCR no lleva registros o análisis de los índices relacionados con la calidad de la investigación, es decir, competitividad investigadora y esfuerzo investigador, por lo que fue necesario construirlos, cuidando de que pudieran ser comparables con los resultados obtenidos por Aceytuno y Paz, (2008).

Respecto del índice competitividad investigadora en la cual se miden proyectos aprobados sobre los presentados, el ITCR no llevaba registro al momento de realizar esta investigación, por lo que no fue posible realizar este análisis y compararlo con la Universidad de Huelva.

Respecto del índice esfuerzo investigador, se pudo constatar que ha existido un crecimiento marginal promedio anual de 5,6 proyectos nuevos de investigación aprobados y de 11,44 académicos que se han agregado a la labor investigativa - 0,49 proyectos por cada nuevo investigador-, teniendo que se pasa de 70 proyectos aprobados y 92 investigadores participantes en el año 2000 , a 120 proyectos aprobados y 195 investigadores participantes en el año 2009.

El comportamiento marginal en la cantidad de investigadores y en los proyectos de inves- tigación ha sido irregular, con algunas caídas entre los años 2004 y 2007, concordando con caídas en los ingresos externos para financiar la investigación. A partir del año 2007, el comportamiento marginal, tanto en cantidad de investigadores como en proyectos de investigación, tiende a recuperarse mostrando una tendencia marginal creciente positiva.

Al analizar el esfuerzo investigador y su comportamiento marginal durante los años 2000 al 2009, no se llegó a una relación de uno a uno -un proyecto de investigación por investigador-, pues en promedio se han presentado 0,70 proyectos de investigación por investigador, indicando que la cantidad de investigadores es superior a la cantidad de proyectos aprobados.

Por otra parte, analizando el comportamiento de este índice, para determinar si ha tenido un comportamiento creciente o decreciente, el análisis muestra que el comportamiento marginal del esfuerzo investigador ha sido decreciente, dado que se pasó de 0.70 proyectos por investigador en el 2000 a 0.62 en el 2009. El ITCR y, en general, las universidades costarricenses no cuentan con estos índices por lo que no es posible hacer alguna comparación entre estas y con la Universidad de Huelva.

Según Aceytuno \& Paz (2008), la calidad de la investigación es un factor especialmente determinante, ya que no tiene sentido la creación de una empresa para explotar resultados de investigación que no presenta suficiente calidad; incluso en el caso de que la empresa llegara a crearse, tendría pocas posibilidades de desarrollo en el futuro y su capacidad de generar crecimiento económico en el territorio sería reducida. Desde esta perspectiva y para que el ITCR presente mejores condiciones para la creación de spin off académicas, es necesario que mejore estos índices en los años venideros.

\subsubsection{Estrategia de apoyo a la generación de spin-off de la universidad}

En el caso del ITCR no existe una estrategia de apoyo como tal, aunque hay esfuerzos en ese sentido, tales como el análisis de la vía legal para implementar las denominadas empresas auxiliares, el contar con una in- 
cubadora de empresas de base tecnológica y múltiples instrumentos para facilitar la vinculación universidad-empresa, tales como la práctica de especialidad como requisito de graduación a nivel de los programas de bachillerato, la venta de servicios a través de Fundatec, el Centro de Vinculación Universidad Empresa, el concurso emprendedor, la incubadora de empresas, entre otros. Desde esta perspectiva, se puede decir que el ITCR tiene una estructura administrativa y académica abundante, para estructurar una estrategia de apoyo a la generación de spin off académicas.

\subsection{Determinantes institucionales}

Según 0`Shea et al., (2004), Djokovic y Souitaris (2008) y Martinelli et al., (2008), los principales determinantes institucionales de la generación de spin-off académicas son: la misión de la universidad, su cultura e historia y la tradición. De acuerdo con las aportaciones revisadas, las universidades cuya misión, cultura o tradición se han dirigido en mayor medida a la creación de empresas o a la colaboración con ellas, presentan una mayor propensión a crear spin-off universitarias como mecanismo de comercialización de los resultados de la investigación.

La misión del ITCR promueve el acercamiento con el sector productivo, lo que se refleja en su tradición y vocación de servicio hacia el sector productivo en particular y hacia la sociedad en general. Como respuesta a esta misión, impuesta en su ley de creación, se han creado diferentes mecanismos de vinculación, siendo el más importante la Fundación Tecnológica de Costa Rica (FundaTEC), la cual administra los recursos financieros, de los proyectos productivos adscritos a las escuelas académicas, lo que ha contribuido a la promoción de una cultura que favorece el emprendimiento interno, al facilitar que los académicos brinden servicios al sector productivo, generando una importante dinamización de los servicios y de los académicos involucrados, produciendo además una importante cantidad de recursos financieros orientados a apoyar proyectos de investigación.

La cultura, historia y tradición del ITCR, están íntimamente ligados con la misión del ITCR y han estado enfocados a promover su vinculación con el sector empresarial a fin de satisfacer las necesidades de éste, siendo la primera la promoción e implementación del programa de prácticas de especialidad de los estudiantes, la cual se lleva a cabo al final de los programas de bachillerato y se hace en una empresa, con el apoyo constante de un docente. Esto promovió un vínculo natural entre las escuelas académicas y las empresas, de tal manera que se brindan servicios contratados, lo que evolucionó en la formación y fortalecimiento de una cultura de emprendedurismo, la cual ha ido creciendo a través de los años. Sin embargo, la cultura de creación de spin-off académicas en el ITCR aún no se encuentra desarrollada y los investigadores no consideran la creación de este tipo de empresas, como una opción viable para explotar comercialmente los resultados de su investigación.

\subsection{Determinantes externos 0 ambientales}

El primer determinante externo es la presencia y cercanía de empresas de capital riesgo, las cuales existen en Costa Rica, pero los fondos de capital de riesgo que manejan son de carácter privado (Macaya Trejos \& Cruz Molina, 2006; Mayorga, 2010). La probabilidad de que una empresa reciba financiamiento procedente de capital de riesgo se reduce a medida que aumenta la distancia geográfica entre ambas empresas (Sorenson \& Stuart, 2001 ). Dado el tamaño de Costa Rica y su infraestructura bancaria, se considera que el aspecto geográfico no es una limitante para acceder, en un futuro, a los denominados capitales de riesgo. La no disponibilidad de capitales de riesgo que apoyen la creación de spin-off universitarias, no es favorable para el surgimiento de spin off académicas en el ITCR.

El segundo determinante externo corresponde a la legislación vigente que afecta a las spin-off universitarias, la que puede constituirse en un freno o en un estímulo a la creación de este tipo de empresas (Golfard \& Henrekson, 2003; Shane, 2004; Bacchiocchi \& Montobbio, 2007 ). En el caso del Instituto Tecnológico de Costa Rica, existe legislación en dos niveles: uno institucional y otro nacional. El primero corresponde a la Ley Orgánica de creación del Instituto Tecnológico de Costa Rica. El segundo promulgado por el Ministerio 
de Ciencia y Tecnología, denominado "Ley para la promoción del desarrollo científico y tecnológico". A nivel nacional, la legislación estipula que las universidades estatales no están facultadas para hacer transferencias de fondos públicos a entes privados. A nivel institucional, la Ley Orgánica del ITCR, faculta la creación de empresas adscritas al ITCR, lo que quiere decir que serían empresas que manejan fondos públicos, lo que difiere del espíritu de lo que constituye una spin-off académica. En contraste con la legislación estadounidense y española, la legislación costarricense es muy diferente, dado que el marco legal del país y la Ley Orgánica del ITCR no favorecen la creación de spin-off académicas.

El último determinante externo señalado es el contexto tecnológico y empresarial que rodea a la universidad. Las universidades situadas en contextos altamente tecnológicos y emprendedores generan un mayor número de spin-off, en comparación con aquellas situadas en entornos menos favorecedores (Roberts, 1991; Saxenian, 1994; Jong, 2006; O'Shea, et al., 2007). Aunque el ámbito de acción del ITCR es a nivel nacional, el contexto empresarial y tecnológico inmediato es el de la provincia de Cartago, donde está ubicada su sede central, el cual no es especialmente propicio para la creación de spin-off, por lo siguiente: el tejido empresarial de Costa Rica está compuesto por un $72 \%$ de microempresas, un $22 \%$ de pequeñas empresas, un $4 \%$ de empresas medianas y un $2 \%$ de grandes. La actividad de las empresas costarricenses se encuentra distribuida de la siguiente manera: el $8 \%$ se ubica en la industria, un $10 \%$ en agricultura un $28 \%$ en comercio y el $55 \%$ en servicios, y distribuido por provincias se encuentra que la provincia de Cartago, representa el $6.8 \%$ de todo el tejido empresarial en comparación con provincias como San José que aglutina un $44,29 \%$, Alajuela un $17,41 \%$, Heredia $10,12 \%$, donde se encuentras los principales Parques Industriales del país. Siendo incluso superada por provincias costeras como Puntarenas con un $8,80 \%$, Guanacaste $7,49 \%$ y ligeramente superior a la provincia de Limón un 5,01\%.

\section{Conclusiones}

Esta investigación hace un aporte importante al identificar spin off académicas del ITCR y la caracterización de los factores de- terminantes en la creación de éstas. Se trata del primer esfuerzo realizado en este campo a nivel costarricense, obteniéndose como conclusión general de este estudio, la falta de conocimiento y comprensión por parte de las autoridades universitarias y de gobierno, de este fenómeno tan importante para el desarrollo del país y la calidad de vida de la sociedad costarricense.

El método de caso utilizado, se fundamenta en técnicas cualitativas para la obtención de información, de carácter no estructurada, flexible y de tipo psicológico y sociológico; utilizando muestras reducidas de las que se obtiene abundante conocimiento. Evidentemente, los resultados obtenidos no son cuantificables ni extrapolables al conjunto total de una población y su utilidad radica en su capacidad para la descripción de hechos y en cómo consigue explicar sus motivaciones con datos (Martínez, 2006).

En cuanto a los factores determinantes en la creación de spin-off académicas, las conclusiones relevantes son:

Sobre los recursos organizativos de la universidad tenemos que a) En cuanto al nivel y naturaleza de los fondos utilizados para financiar la investigación, el ITCR requiere hacer un mayor esfuerzo en la búsqueda de fondos externos para el apoyo de la investigación, ya que en la actualidad el aporte externo representa un quinto de la inversión pública, lo cual no se puede calificar como positivo, comparado con datos de universidades españolas, con valores por encima del $50 \%$. b) La calidad de la investigación en el ITCR, desglosada en dos elementos diferentes e igualmente importantes: i) Calidad de los investigadores. Dado los bajos porcentajes encontrados, con respecto a la cantidad de proyectos nuevos por año y de investigadores participantes, al comportamiento marginal irregular encontrado entre los años 2000 y 2009 , a la caída en la cantidad de proyectos de investigación nuevos presentados entre los años 2004 y 2007 , al bajo crecimiento de 0,49 proyectos por cada nuevo investigador, se puede concluir que deben de mejorarse estos aspectos, en conjunto, para favorecer la aparición de spin-off universitarias en el ITCR; ii) Esfuerzo investigador. Los datos recopilados muestran una baja relación entre cantidad de proyectos de investigación presentados y 
cantidad de investigadores participantes, correspondiente a un promedio de 0,70 del 2000 al 2009, con un comportamiento decreciente a través de los años, indicando que aunque la cantidad de investigadores ha aumentado, la cantidad de proyectos de investigación presentados ha disminuido, por lo que debe incentivarse la generación de una mayor cantidad de proyectos de investigación, para favorecer la creación de spin-off en el ITCR; c) Estrategia de apoyo a la generación de spinoff de la universidad. Se constató que en el ITCR no existe una estrategia de apoyo a la generación de spin-off, pero que existe una fuerte infraestructura para apoyar la creación de una, por lo que se concluye que sería necesario articular todos estos esfuerzos a través de la creación de una estrategia, de manera que se favorezca la creación de spin-off.

Sobre los determinantes institucionales para la generación de spin-off, los cuales han sido identificados en la literatura como la misión de la universidad, su cultura e historia y la tradición, tenemos que en el ITCR se ha promovido un acercamiento con el sector productivo, desde sus orígenes en el año 1977, creándose y fortaleciéndose a través del tiempo una cultura y tradición de servicio a este sector, lo que constituye un aspecto positivo que apoya la creación de spin-off académicas. El ITCR ha avanzado en el paradigma de la universidad emprendedora y del emprendedor académico, a través de varios instrumentos de vinculación, como son la FundaTEC y la incubadora de empresas, lo que constituye un estímulo para la creación de spin-off académicas. A pesar de estos aspectos positivos, se concluye que la cultura de creación de empresas en el ITCR aún no se encuentra desarrollada, principalmente debido a la falta de articulación de todos estos esfuerzos para apoyar la creación de spin-off y a que los investigadores no consideran la creación de spin-off como una opción viable para explotar comercialmente los resultados de sus investigaciones.

En cuanto a los determinantes externos o ambientales, dentro de los cuales están empresas de capital de riesgo, la legislación vigente y el contexto tecnológico y empresarial que rodea a la universidad, tenemos a) Empresas de capital de riesgo. Dado que este tipo de empresas existen en Costa Rica pero con fondos de carácter privado, que no se pueden ofrecer públicamente, se puede concluir que la carencia de empresas de capital de riesgo es un impedimento para el estímulo y realización de los proyectos de spin-off que surgieren en el ITCR; b) La legislación vigente. El marco legal del país y la ley Orgánica del ITCR no contemplan dentro de su normativa el traslado de fondos públicos a iniciativas privadas, por lo que se puede concluir que la legislación institucional y nacional son una limitación para la creación de spin-off académicas; c) Los contextos tecnológico y empresarial que rodea a la universidad. Dada la baja dinámica tecnológica y económica de la provincia de Cartago, donde se encuentra ubicado la sede central del ITCR, se puede concluir que es a escala nacional donde los contextos tecnológico y empresarial se han constituido en un factor positivo para la creación de spin-off académicas.

El ITCR posee fortalezas y debilidades desde la perspectiva de los factores determinantes de la creación de spin off académicas, no obstante, gracias al cumplimiento de su misión, a la incursión de este en el sector productivo, a través de las prácticas de especialidad, la venta de servicios y la investigación contratada hacen de esta institución, así como también la existencia de un sector productivo que demanda y ha encontrado cobijo a sus necesidades en el ITCR, es que se puede concluir que esta universidad posee fortaleza natural para la creación de spin off, por lo que requiere de legislación moderna, empresas de capital semilla, incrementar la atracción de fondos externos y, ante todo, fortalecer la calidad de la investigación, es decir, mejorar el planteamiento de propuestas de investigación, mayor cantidad de proyectos de investigación por investigador y por último, plantear una estrategia Institucional que abarque los ámbitos administrativo, docente e investigativo para articular todos los esfuerzos del ITCR parar la promoción de spin off académicas.

Los resultados de esta investigación pretenden brindar pautas a la academia universitaria latinoamericana y a los tomadores de decisiones relacionados con la universidad pública, acerca de los determinantes que favorecen el proceso de creación de spin-offs. Pretende proporcionar ideas a la academia, para que sus actividades de promoción y divulgación sobre creación de spin-off, tengan 
un mayor impacto al interior de la comunidad universitaria y sirva de referente para fortalecer una cultura donde se ofrezca una mayor transferencia de conocimiento a la sociedad. A las entidades públicas y privadas de fomento a la actividad empresarial, les ofrece nuevas perspectivas, respecto de la importancia de reforzar sus programas de intervención para impulsar una mayor imbricación de la Universidad en el tejido empresarial de la Región.

\section{Referencias}

Aceytuno, M., y Paz, M. (2008). La creación de spin-off universitarias: el caso de la Universidad de Huelva. Economía industrial, 368, pp. 97-111.

ANCES. (2003). La Creación de Empresas de Base Tecnológica. Una experiencia práctica Proyecto para la promoción de empresas innovadoras de base tecnológica. Valencia, España: ANCES, CEIN y otros.

Bacchiocchi, E. y Montobbio, F. (2007). Knowledge Diffusion from University and Public Research. A Comparison between US Japan and Europe using Patent Citations. Journal of technology transfer, Springer 34 (2) pp. 169-181, Online First. doi: 10.1007/ s10961-007-9070-y.

Beraza, J. M. y Rodríguez, A. (2010). Factores determinantes de la utilización de las spin-off como mecanísmo de transferencia de conocimiento en las universidades. Investigaciones Europeas de Dirección y Economía de la Empresa, 16(2), pp. 115-135.

Blumenthal, D., Campbell, E., Causiono, N. y Louis, K. S. (1996). Participation of Life Science Faculty in research Relationships with Industry. The New England Journal of Medicine, 335(23), pp. 1734-1740.

Carlsson, B. y Fridh, A.-C. (2002). Technology Transfer in United States Universities. A Survey and Statistical Analysis. Journal of Evolutionary Economics, 12, pp. 199-232.

Casanova, J. (2004). La experiencia de los CEEIS Españoles apoyando EIBTS. Paterna. Valencia-España: ANCES, pp. 619-626.
COTEC. (2006). Tecnología e innovación en España. [Informe]. Madrid, España: Informe COTEC 2006.

Debackere, K. (2000). Managing Academic R\&D Business at K.U. Leuven: Context, Structure and Process. R\&D Management, 30(4), pp. 323-328.

Di-Gregorio, D. y Shane, S. (2003). Why do Some Universities Generate More Startups than Others? Research Policy, n. 32, pp. 209-227.

Djokovic, D. y Souitaris, V. (2008). Spinouts from academic institutions: a literature review with suggestions for further research. The Journal of Technology Transfer, 33(3), pp. 225-247. doi: 10.1007/s10961-0069000-4.

Etzkowitz, H. y Leydesdorff, L. (2000). The dynamics of innovation: from National Systems and "Mode 2" to a Triple Helix of university-industry-government relations. Research Policy, 29 (2), pp. 109-123. doi: 10.1016/So048-7333(99)00055-4.

Feldman, M. P., Feller, I., Bercowitz, J. y Burton, R. (2002). Equity and the Technology Transfer: Strategies of American Research Universities. Management Science, 48( 1), pp. 90-104.

Florida, R. R., y Kenney, M. (1988). Venture Capital-Financed Innovation and Technological Change in the USA. Research Policy, m. 17, pp. 119-137.

Gaceta. (1999). Ley Orgánica del Instituto Tecnológico de Costa Rica en C. R. Leyes, d. (Ed.), Ley 4777. San José, Costa Rica: Asamblea Legislativa: publicada en el Diario Oficial La Gaceta del 10 de junio de 1971.

Golfard, B. y Henrekson, M. (2003). Bottomup versus Top-down Policies towards the Commercialization of University Intellectual Property. Research Policy, n. 32, pp. 639-658.

Henrekson, M. y Rosenberg, N. (2001). Designing Efficient Institutions for ScienceBased Entrepreneurship: Lesson from the US and Sweden. Journal of technology transfer, 26(3), pp. 207-231. 
Jain, S. y George, G. (2007). Technology Transfer Offices as Institutional Entrepreneurs: The Case of Winconsin Alumni Research Foundation and Human Embrionic Stem Cells. Industrial and Corporate Change, 16(4), pp. 535-567.

Jong, S. (2006). How Organizational Structures in Science Shape Spin-off Firms: The Biochemistry departments of Berkeley, Stanford, and UCSF and the Birht of the Biotech Industry. Industrial and Corporate Change, 15(2), pp. 251-283.

Kenney, M. y Goe, R. (2004). The Role of Social Embeddedness in Professorial Entrepreneurship: A Comparison of Electrical Engineering and Computer Science at UC Berkeley and Stanford. Research Policy, n. 33, pp. 691-707.

Kirby, D. (2006). Creating Entrepreneurial Universities in the UK: Applying Entrepreneurship Theory to Practice. The Journal of Technology Transfer, 31(5), pp. 599-603. doi: 10.1007/s10961-006-9061-4.

Laborda, F. y Briones, A. (2010). Innovación y Tecnología Factores de dirección estratégica de los agronegocios en Costa Rica y la Región de Murcia, pp. 99-116. Costa Rica-España: Universidad Politécnica de Cartagena.

Ley Orgánica 4/2007. (2001) (Vol. por la que se modifica la Ley Orgánica 6/2001, de 21 de diciembre, de Universidades): BOE n- 89 de 13 de abril de 2007.

Link, A. N. y Scott, J. T. (2005). Opening the ivory tower's door: An analysis of the determinants of the formation of U.S. university spin-off companies. Research Policy, 34(7), pp. 1106-1112. doi: DOI: 10.1016/j.respol.2005.05.015.

Lockett, A., Siegel, D., Wright, M. y Ensley, $M$. (2005). The creation of spin-off firms at public research institutions: Managerial and policy implications. Research Policy, 34(7), pp. 981-993. DOI: 10.1016/j.respol.2005.05.010

Lockett, A. y Wright, M. (2005). Resources, Capabilities, Risk Capital and the Creation of University Spin-out Companies. Research Policy, 34, pp. 1043-1057.
Louis, K., Blumenthal, D., M.Gluck y Stoto, M. (1989). Entrepreneurs in Academe: Behaviours among Life Scientist. Administrative Science Quarterly, 34(1), pp. 110-131.

Macaya, G. y Cruz, A. (2006). Proyecto Estrategia Siglo XXI Visión de la ciencia y la tecnología en Costa Rica: una construcción colectiva. San José, Costa Rica: Fundación Costa Rica- Estados Unidos de América para la Cooperación.

Markman, G. D., Gianiodis, P. T. y Phan, P. (2008). Full-Time Faculty or Part-Time Entrepreneurs. IEE Transactions on Engineering Management, 55(1), pp. 29-36.

Martinelli, A., Meyer, M. y Von-Tunzelmann, N. (2008). Becoming an entrepreneurial university? A case study of knowledge exchange relationships and faculty attitudes in a medium-sized, research-oriented university. The Journal of Technology Transfer, 33(3), pp. 259-283. Doi: 10.1007/s10961-007-9031-5.

Martínez, P. C. (2006). El método de estudio de caso estrategia metodológica de la investigación científica. Pensamiento \& gestión, n. 20, pp. 165-193.

Mayorga, G. (2010, 09-02). Instrumento financiero permitirá al público invertir en capital de riesgo: Nacerá nueva figura de inversión en fondos. La Nación.

Meseri, O. y Maital, S. (2001). A Survey Analysis of University-Technology Transfer in Israel: Evaluation of Projects and Determinants of Success. Journal of technology transfer, $\mathrm{n}$. 26, pp. 115- 126.

Nlemvo, F., Pirnay, F. y Surlemont, B. (2002). A stage model of academic spin-off creation. Technovation, 22(5), pp. 281-289. doi: DOI: 10.1016/S0166-4972(01)00019-0.

O'Gorman, C., Byrne, O. y Pandya, D. (2008). How Scientists Commercialise New Knowledge via Entrepreneurship? Journal of technology transfer, 33(1), pp. 22-34.

O'Shea, R. y Allen, T. (2005). Determinants and consequences of university spin off activity: a conceptual framework. Paper presented at the Babson- Kauffman Entrepreneurship Conference. 
O'Shea, R., Allen, T. y Morse, K. (2005). Creating the entrepreneurial university: the case of MIT. Paper presented at the Presented at Academy of Management Conference, Hawaii.

O'Shea, R., Allen, T., Morse, K., O’Gorman, C. y Roche, F. (2007). Delineating the anatomy of an entrepreneurial university: the Massachusetts Institute of Technology experience. [Delineando la anatomía de un emprendedor universitario: La experiencia del Instituto Tecnológico de Massachusetts]. R\&D Management, 37(1), p. 16.

O’Shea, R., Allen, T., Morse, K., O’Gorman, C., Roche, F. y Cáceres, R. (2004). Universities and Technology Transfer: A review of academic entrepreneurship literature. A Review of academic Entrepreneurship Literature. Irish Journal of Management, 25 (2), pp. 11-29.

O'Shea, R., Chugh, H. y Allen, T. (2008). Determinants and consequences of university spinoff activity: a conceptual framework. The Journal of Technology Transfer, 33(6), pp. 653-666. [10.1007/s10961-007-9060-o]

Parker, D. D., y Zilberman, D. (1993). University Technology Transfer: Impacts on Local and US Economies. Contemporary Policy Issues, 11 (2), pp. 87-100.

Powers, D. B. y McDougall, P. P. (2005 ). University Start-up Formation and Technology Licensing with Firms that Go Public: A Resource-based view of Academic Entrepreneurship. Journal of Business Venturing, n. 20, pp. 291-311.

Queralt, L. (2008). Trayectorias Laborales y Personales de Mujeres profesionales de carreras tradicionalmente masculinas: El caso de las egresadas del Instituto Tecnológico de Costa Rica. Tesis de Máster en Género y Políticas de Igualdad. Valencia, España: Universidad de Valencia.

Ramón, T. y García, D. (2004). El plan de negocio en la toma de decisiones del capital riesgo en España. Harvard Deusto Finanzas y Contabilidad, n. 58, pp. 60-67.

Ramón, T. y García, D. (2006). La participación financiera del capital de riesgo español según la procedencia pública o privada de sus recursos. Economía industrial, n. 362, pp. $155-164$.

Rasmussen, E., Moen, Ø. y Gulbrandsen, M. (2006). Initiatives to promote commercialization of university knowledge. Technovation, 26(4), pp. 518-533. [doi: DOI: 10.1016/j.Technovation.2004.11.005]

Roberts, E. B. (1991). Entrepreneurs in High Technology Lesson from MIT and Beyond. New York, Oxford: Oxford University Press.

Roberts, E. B. y Malone, D. E. (1995). Policies and Structures for Spinning Off New Companies from Research and Development Organizations. Working Paper Series Alfred P. Sloan School of Management, Massachusetts Institute of Technology.

Saxenian, A. (1994). Regional Advantage: Culture and Competition in Silicon Valley and Route 128. Cambridge (Mass.) y Londres.

Shane, S. (2004). Academic Entrepreneurship University spin offs and Wealth Creation. Londres, R.U.: Edward Elgar Publishing.

Siegel, D. S. y Phan, P. H. (2006). The Effectiveness of University Technology Transfer. Foundations and Trends in Entrepreneurship. 2(2).

Siegel, D. S., Waldman, D. y Link, A. (2003). Assessing the Impact of Organizational Practices on the Relative Productivity of University Technology Transfer Offices: An Exploratoy Study. Research Policy, n. 32, pp. 27-48.

Sorenson, O. y Stuart, T. E. (2001). Syndication Network and the Spatial Distribution of Venture Capital Investments. American Journal of Sociology, 106 (6), pp. 1546-1588.

Stuart, T. E. y Ding, W. W. (2006). When do Scientists Become Entrepreneurs? The Social Structural Antecedents of Commercial Activity in the Academic Life Sciences. American Journal of Sociology, 112(1), pp. 97-144.

Wright, M., Birley, S. y Mosey, S. (2004). Entrepreneurship and University Technology Transfer. Journal of technology transfer, n. 29, pp. 235- 246. 
Wright, M., Hmieleski, K. M., Siegel, D. S. y Ensley, M. D. (2007). The role of human capital in technological entrepreneurship. Entrepreneurship: Theory and Practice, 31(6), pp. 791-806. [doi: 10.1111/j.15406520.2007.00202.x.]

Yin, R. (2009). Discovering the future of the case study method in evaluation research. Evaluation Practice, 15(3), pp. 283-290. [doi: Doi: 10.1016/08861633(94)90023-x.]

Zucker, L. G., Darby, M. R. y Brewer, M. B. (1998). Intellectual Human Capital and the Birth of US Biotechnology Enterprises. The American Economic Review, n. 88, pp. 290306. 